Original Article

\title{
Therapeutic Efficacy and Safety of Dihydroartemisinin-Piperaquine (DP) for the Treatment of Uncomplicated Plasmodium Vivax Malaria: A Single Center Study
}

\author{
Salma Shaikh, Imran Ahmed, Sikander Munir Memon, Abdul Saleem, \\ Hafeezullah Memon, Ayaz Babar
}

ABSTRACT

BACKGROUND: Ninety-five millions of Pakistan's 161 million people, roughly $60 \%$ of Pakistan's population live in malaria endemic regions. Despite a well-established malaria control programme, $\mathbf{5 0 0 , 0 0 0}$ malaria infections and 50,000 malaria-attributable deaths occur each year in Pakistan. In Pakistan $15 \%$ population lives in high transmission area, $84 \%$ in low transmission and $1 \%$ in malaria free area, with $64 \%$ vivax and $36 \%$ Falciparum infections.

OBJECTIVE: The objective of this study was to assess the therapeutic efficacy and safety of Dihydroartemisinin-piperaquine (DP) for the treatment of uncomplicated Plasmodium vivax malaria in subjects.

METHODS: Its an observational study, conducted at Outpatient Department of Liaquat University Hospital Hyderabad, from December 2012 to December 2013. World Health Organization (WHO) standard protocol for efficacy studies (open-labelled clinical trial) was followed. The subjects with fever or history of fever for $\mathbf{4 8}$ hours aged between $\mathbf{6}$ months to 15 years with microscopically confirmed uncomplicated $P$. vivax infection were included. Total 109 patients fulfilled the inclusion criteria. Out of 109 patients, 103 had completed the study. Patients were treated with Dihydroartemisinin-piperaquine over three days. Clinical and parasitological parameters were monitored over a 42-days follow-up period to evaluate drug therapeutic efficacy.

RESULTS: Adequate clinical and parasitological response of treatment (ACPR) for Dihydroartemisininpiperaquine (DP) was seen in $102 / 103(99.02 \%)$ patients, no early or late clinical failure was seen while late parasitological failure was seen on $21^{\text {st }}$ day in one patient. No adverse events were reported.

CONCLUSION: Dihydroartemisinin-piperaquine is safe and effective treatment option for uncomplicated vivax malaria.

KEYWORDS: Dihydroartemisinin-piperaquine (DP). Chloroquine (CQ), Early Treatment Failure (ETF), Late Clinical Failure (LCF), Adequate Clinical and Parasitological Response (ACPR).

This article may be cited as: Shaikh S, Ahmed I, Memon SM, Saleem A, Memon H, Babar A. Therapeutic Efficacy and Safety of Dihydroartemisinin-Piperaquine (DP) for the Treatment of Uncomplicated Plasmodium Vivax Malaria: A Single Center Study. J Liaquat Uni Med Health Sci. 2017;16(02):93-8. doi: 10.22442/jlumhs.171620513

\section{INTRODUCTION}

Approximately $42 \%$ of the worldwide population is at risk of $P$. vivax infection. Seven countries in Asia (China, Indonesia, Myanmar, Pakistan, the Philippines, Thailand and Vietnam) are among the main ten countries with most of their population at risk of P. vivax ${ }^{1}$. According to $\mathrm{WHO}$ world malaria report 2011 the targets of roll back malaria program of reducing mortality by $50 \%$ were not achieved by 2010 , but there has been significant reduction in cases by $17 \%$ and mortality by $26 \%$ worldwide. Pakistan is among five countries out of 23 in EMRO which has shown no significant decline from 2000 to 2010 . 22\% of 1.2 million confirmed cases in this region were reported from Pakistan ${ }^{2}$.

Ninety-five millions of Pakistan's 161 million people, roughly $60 \%$ of Pakistan's population live in malaria endemic regions. After eradication efforts in the 1960's, malaria surged back to an epidemic level in the 1970s. In recent years, an uplift in malaria can be partially attributed to flood that affected approximately 20 million people in over 60 districts $^{3}$. Despite a well-established malaria control programme, 500,000 malaria infections and 50,000 malaria-attributable deaths occur each year in Pakistan ${ }^{4}$.

According to WHO world malaria report 2011 the targets of roll back malaria programme of reducing mortality by $50 \%$ were not achieved by 2010 , but there has been significant reduction in cases by $17 \%$ and mortality by $26 \%$ worldwide 5 . Pakistan is among five countries out of 23 in EMRO which has shown no significant decline from 2000 to 2010. 22\% of 1.2 million confirmed cases in this region were reported 
from Pakistan ${ }^{5}$.

In Pakistan $15 \%$ population lives in high transmission area, $84 \%$ in low transmission and $1 \%$ in malaria free area, with $64 \%$ vivax and $36 \%$ Falciparum infections ${ }^{6}$.

$P$. vivax is a larger and more serious threat than previously estimated. Recent studies have shown that $P$. vivax can result in a severe disease that resembles the cerebral malaria caused by $P$. falciparum ${ }^{8}$. In addition, the lifecycle of $P$. vivax parasites includes a dormant stage that is difficult to detect and treat. Treatment failure due to resistance to chloroquine (CQ) in P. vivax was first documented in 1989 among Australians repatriated from Papua New Guinea ${ }^{9}$. Since then, sporadic resistance to $C Q$ has been reported from other countries including Brazil, Ethiopia, Myanmar and Turkey. As such, the recent documented emergence of resistance to $C Q$ in vivax malaria deserves paying attention to $P$. vivax drug sensitivity. Early diagnosis followed by prompt and effective treatment remains a cornerstone for the reduction of malaria-related morbidity and mortality ${ }^{10}$. Resistance to ACT monitoring of therapeutic efficacy of antimalarial medicine is essential every 2 years ${ }^{11}$. According to world malaria report 2016, children aged under 5 years with fever should be sought from a professional or trained provider ${ }^{12}$. Along this thread, alternative antimalarial treatments for $P$. vivax are needed. This drug has been used in Mimiko, Papua since 2006 and was subsequently recommended for use in Papua in 2009, based on a 2004 Mimika study. DP has been shown to be effective and safe with good patient compliance for treatment of uncomplicated P. falciparum \& vivax malaria in Papua, as well as in other countries. However, limited data on the use of DP in Asian children is available. Dihydroartemisinin-piperaquine has been approved by FDA as first line of drug ${ }^{13-14}$. To provide supporting evidence based data for wide scale use of DP, further evaluation of its efficacy in children as a vulnerable group outside of Pupa, is needed. So objectives of this study was to measure therapeutic efficacy and safety of Dihydroartemisinin-piperaquine to treat uncomplicated vivax malaria by determining the proportion with early treatment failure, late clinical failure, late parasitological failure or an adequate clinical and parasitological response as indicators of efficacy, to determine recrudescence from new infection by PCR analysis. The results of this study will provide guidance to the national control programs to develop and update their case management policies and guidelines.

\section{METHODOLOGY}

This trial was conducted at Outpatient Department of Liaquat University Hospital Hyderabad, from December 2012 to December 2013. The patients attending pediatric clinic were screened and included in the study if they fulfilled the following inclusion criteria: Age between 6 months to 15 years, mono-infection with P.vivax confirmed by microscopy, parasitaemia of $1000-250000 / \mu$ l asexual forms and axillary temperature $>37.5{ }^{\circ} \mathrm{C}$ or history of fever during the past 48hours, ability to swallow oral medication, willingness of parents to enroll their child in study and sign the consent form.

All patients who had general danger signs (aged $<5$ years) or those who were classified as severe malaria (according to $\mathrm{WHO}$ ), mixed or mono-infection cause by Plasmodium species other than $\mathrm{P}$ vivax, patients who were severely malnourished, presence of other febrile conditions (i.e. bacterial pneumonia, skin infection, ear infection) or any other chronic disease (i.e., congenital heart disease, hepatitis and, T.B), patient on drugs, which may interfere with antimalarial metabolism \& previous history of allergy to drug were excluded from the study.

Therapeutic efficacy will be assessed by returning of normal body temp for $>48 \mathrm{hrs}$ and clearance of trophoziotes for $>48 \mathrm{hrs}$ and safety will be assessed by appearance of unwanted symptoms, i.e. vomiting, jaundice, lethargy and shortness of breath.

Sample Size

LUH patients' data

2013

Total patient screen 2543, +ve 94 (Falcp. 11, vivax 83)

2014

Patient screen 3994, +ve 69 (Falcp. 5, vivax 64)

2015

Patient screen 3189, +ve 32 (Falcp. 6, vivax 26)

Population size (for finite population correction factor or $\mathrm{fpc}$ ) (N): $\quad 9,726$

Hypothesized \% frequency of outcome factor in the population (p): $7 \%+/-5$

Confidence limits as \% of 100 (absolute $+/-\%$ ) (d):5\%

Design effect (for cluster surveys-DEFF): 1

Cl was set $95 \%$ and sample size was calculated 100 .

Equation

Sample size $n=\left[D E F F^{*} N p(1-p)\right] /\left[\left(d 2 / Z 21-\alpha / 2^{*}(N-1)\right.\right.$ $\left.+p^{*}(1-p)\right]$

Study was conducted from December 2012 to December 2013. Total 109 patients fulfilled the inclusion criteria. 103 / 109 patients completed the study. Patients were treated with Dihydroartemisininpiperaquine for 3 days. Clinical and parasitological response was monitored over a 42-days follow-up period to evaluate drug efficacy.

Patients with fever or complain of fever for last 2 days were screened for malaria. The individuals who fulfilled the criteria were included in the study. A 
personnel identification number and treatment was given only when parents or guardians understood and agreed to terms and conditions of study protocol and signed the consent form. Demographic information followed by a complete medical history, thorough physical examination and contact details were then recorded.

Body weight was documented on day 0 , temperature and presence or absence of general danger signs were recorded on each follow-up visit. Axillary temperature was checked at every visit. Dihydroartemisinin-piperaquine was administered at doses recommended by manufacturer [Table I] for three days under supervision.

Children were watched for 30 minutes after antimalarial drug for unwanted effects or vomiting. If child vomited in above time period the dose of same drug was repeated and watched for an extra $30 \mathrm{~min}$. Child was excluded from the study in case of vomiting again and managed with appropriate therapy. Paracetamol was recommended for fever in prescribed measurements where needed.

Primaquine $(0.3 \mathrm{mg}$ of base $/ \mathrm{kg}$ of body weight for 14 days) was prescribed in those children whose age was $>5$ years.

Microscopy was done at the time of screening on day 0 and to confirm adherence to the inclusion criteria. At that point blood films were checked by two trained microscopist separately on days $0,2,3,7,14,21,28$ $\& 42$ or on any time day if the patient returned due to complain of fever and parasitological clearance was assessed.

The thick film for screening was utilized to check exact asexual parasite count and WBC's in a limited number of microscopic fields. The enrolment criteria were 1 parasite/ 6WBCs, which is equal to 1000 asexual parasites/micro Littre.

Parasite density was calculated on second slide by counting asexual parasites per 200 WBCs with a hand count counter. When a field was begun, then it was counted completely. During counting if $>500$ parasites were found before 200 WBCs the counting was stopped. Parasite density was expressed as the number of asexual parasites every $\mu \mathrm{l}$ of blood, was calculated by following formula

Parasite density $($ per $\mu \mathrm{l})=$ number of parasites counted (6000)

Number of leukocytes counted

The same procedure was utilized to obtain the parasite density on every consequent blood slide. At the point when the number of asexual parasites was < 10 every 200 WBCs in subsequent smears, counting was carried out against 500 WBCs. A blood film was declared negative when there was no any single asexual parasite was found against 1000 WBCs.

Moreover, 100 fields of the second-thick film were checked for mixed infection; if there was any doubt, the thin film was carefully checked for confirmation. If still there was any uncertainty regarding mixed infection the patient was excluded from data after completion of follow -up.

Two well-trained microscopists examined all the slides separately, and parasite densities were computed by averaging the two tallies. Blood smears with different results (if difference in parasite density was $>50 \%$ ) was reconsidered by a third microscopist, and parasite density was computed by averaging the two nearest tallies.

The basic follow-up schedule was 8 visits for each patient. Two separate case study Performa were used, one for recording general data \& clinical assessment and other for unwanted side effect in every child. The visiting day and time was clarified, and a follow up card to every individual with study number was given.

From there on, patients were properly examined clinically and blood slides for parasite density were prepared on days 2, 3 and 7 and afterwards every week for the rest of the follow-up period, i.e. on days 14, 21, 28 \& 42. Patients were encouraged to come any time for amid the subsequent period if symptoms return. Specifically, caretakers were told to bring their child to the health facility whenever if any signs of severe disease appeared (i.e. refusal to feed, continuous vomiting, fits, not able to sit or stand). On each follow up visit patient was evaluated for potential treatment failure and unwanted serious effect of drug.

Patients who did not visit on day 1 or 2 were withdrawn from the study. Others were encouraged to come to the center for follow up and those who did not come were followed up at their home.

Kaplan Meier analysis was used for data management and analysis. Data was analyzed by two methods: The Kaplan-Meier method and per-protocol analysis.

\section{RESULTS}

\section{Efficacy}

Study started on 22-12-2012 and was completed on 21-12-2013 total 1324 patients were screened. $195 / 4324(4.5 \%)$ had MP +ve among these 132 had Plasmodium vivax, 24 had mixed infection 39 had falciprum.109 patients who fulfilled inclusion criteria were enlisted in the study while others were treated according to national recommendation. 103 out of 109 enrolled completed the study, while 5 patients were withdrawn and 1 was lost to follow up. No early or late clinical failure was seen but one patient had 
reappearance of trophozoites on day 21, with ACPR of $99.02 \%$ [Fig 1]. At 24 hours the parasite clearance was $99 \%$. The gametocytes were seen in 89 , by day 7 the clearance rate was $100 \%$ [Table I]

Safety: Only three patients vomited the $1^{\text {st }}$ dose on day 0 but tolerated well when dose was repeated. One patient was withdrawn due to persistent vomiting after repeat dose. There were no reports of adverse effects on follow up.

The gender ratio was Male $63(61 \%)$ and Female 40 (39\%). Male \& Female ratio was 1.5: 1 . Regarding age variation, patients age $1-5(2.45)$ years were $40(39 \%)$, while age $5-15(8.55)$ years were $63(61 \%)$, while at the time of enrolment $92(89.3 \%)$ had fever while 11 $(10.7 \%)$ were a febrile [Fig 2].

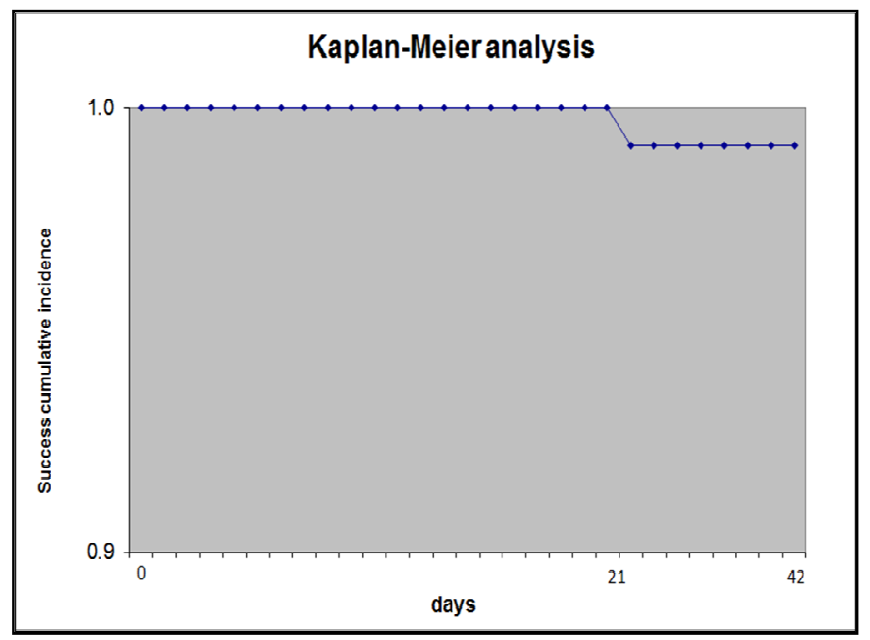

TABLE I: CLINICAL \& PARASITOLOGICAL RESPONSE BY DIHYDROARTEMISININPIPERAQUINE (DP)

\begin{tabular}{|c|c|}
\hline \multicolumn{2}{|c|}{ Pakistan } \\
\hline Fever Clearance (\%) & \\
D1 & $82(89.1 \%)$ \\
D2 & $10(100 \%)$ \\
D3 & - \\
Parasitemia clearance (\%) & $102(99 \%)$ \\
D1 & $103(100 \%)$ \\
D2 & \\
Gametoyte Clearance (\%) & $83(93.3 \%)$ \\
D1 & $89(100 \%)$ \\
D7 & 103 \\
42 Day Follow Up & 00 \\
(Analysis Number) & 00 \\
ETF & 01 \\
LCF & $102(99.02 \%)$ \\
LPF & 00 \\
ACPR & \\
Adverse events & \\
\hline
\end{tabular}

FIGURE I: FLOW CHART OF PATIENTS TREATED WITH DIHYDROARTEMISININ-PIPERAQUINE IN HYDERABAD, SINDH, PAKISTAN

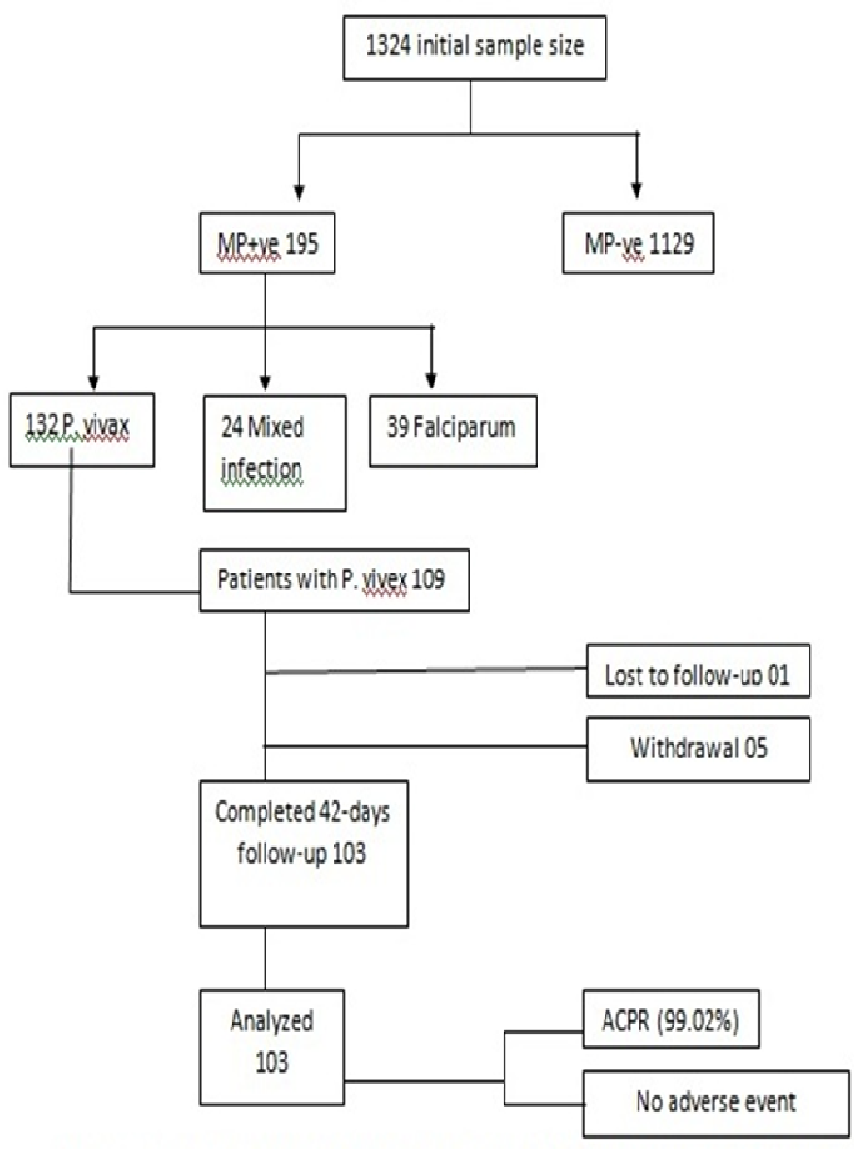

FIGURE 2

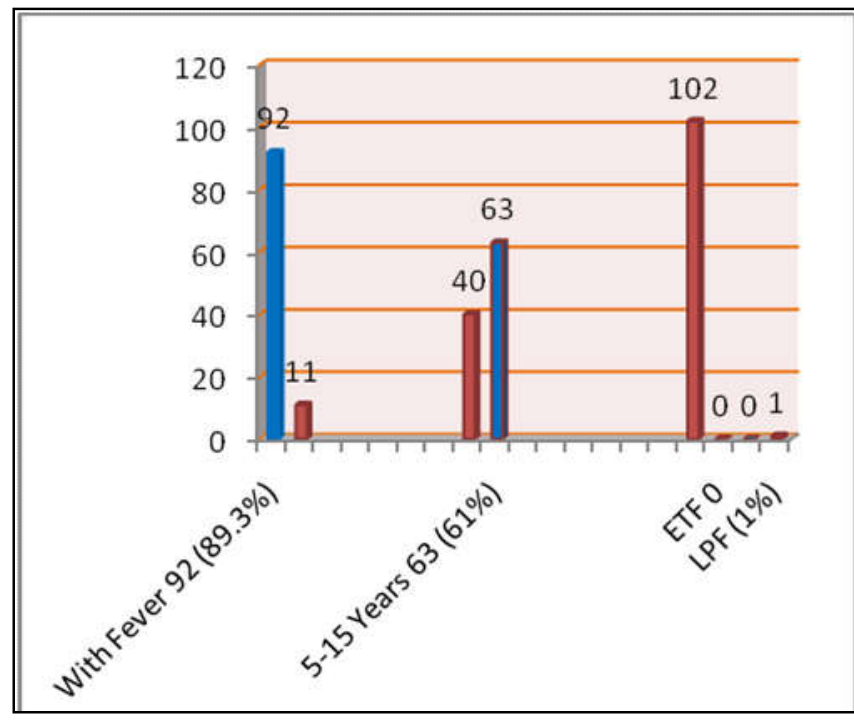

DISCUSSION

Antimalarial drug resistance is the major issue in worldwide control of malaria. Despite WHO 
recommendation of confirming malaria case before treatment, majority of cases in both public and private sector are treated on clinical suspicion. Although Chloroquine is still effective for plasmodium vivax, resistance is being reported from different countries with increasing vivax cases in Sindh. Dihydroartemisinin-piperaquine DP comes as a fixed \& single dose regimen for 3 days of administration. This drug has been used in Mimika, Papua since 2006 and was subsequently recommended for use in Papua in 2009, based on a 2004 Mimika study. In Afghanistan Between July 2007 and February 2009, an open-label, randomized controlled trial of chloroquine and Dihydroartemisinin-piperaquine had been done which showed that Dihydroartemisinin-piperaquine was superior to chloroquine ${ }^{14}$.

A prospective study was conducted in Indonesia in which two anti-malarial drugs were tested against uncomplicated plasmodium vivax. Which demonstrates $A A Q$ and $D H P$, both combined with $P Q$, were effective for blood-stage parasite clearance of uncomplicated $\mathrm{P}$. vivax malaria. Both medicines were effective, however DHP + PQ were better tolerated ${ }^{15}$.

This study shows efficacy of $99.02 \%$, with parasite clearance of $99 \%$ on day 1 . It was well tolerated with no adverse side effect on 42 days follow up. Results are similar to other trials such as comparative trial of Chloroquine and DAP in Afghanistan ${ }^{16}$ and Open-label, randomized controlled study showing that DAP is more effective than CQ+SP, AST+SP \& AL in treating uncomplicated P.Vivax malaria ${ }^{16}$

Gametocytes were seen in $89(86.4 \%)$ cases on enrolment, but cleared in $83(93.3 \%)$ on day 1 with 89 $(100 \%)$ clearances by day 7 . [Table1], similar effect of DAP was seen on gametocyte clearance in another study ${ }^{17}$.

Primaquine is not indicated for children under five and not prescribed to most children over five due to unavailability in private market or reluctance of physicians to use it due to its potential side effects in G6PD deficient patients which results in recurrent attacks of vivax malaria in children. Anemia in infants and children can leads to poor physical and mental development ${ }^{18}$. Almost $67.7 \%$ children suffer from anemia in Sindh with recurrent vivax malaria being an important contributing factor ${ }^{19}$. As $99.02 \%$ of children treated with DP showed ACPR for 42 days with no relapses this may prove to be an added advantage in treatment \& prevention of anemia.

In view of large private sector in Pakistan it may be useful to use one effective drug for all kinds of malaria which makes national guidelines easier to follow. More studies on efficacy of DP are required for both falciparum and Vivax malaria for recommendation of policy change in future. Currently we must disseminate and implement the guidelines for confirmation of malaria cases and treat them according to national guidelines.

\section{CONCLUSION}

Dihydroartemisinin-piperaquine (DP) is safe and effective therapy for uncomplicated vivax malaria.

\section{ACKNOWLEDGMENTS}

The investigators are grateful to Child welfare Trust \& Hilton pharma for logistic support.

\section{REFERENCES}

1. Guerra CA, Howes RE, Patil AP, Gething PW, Van Boeckel TP, Temperley $\mathrm{WH}$, et al. The International Limits and Population at Risk of Plasmodium vivax Transmission in 2009. PLoS Negl Trop Dis. 2010;4(8):e774.

2. World Health Organization. World Malaria Report 2011 [Internet]. WHO Global Malaria Programme. 2011. Available from: http://www.who.int/malaria / world_malaria_report_2011/ 97892415 $\overline{6} 4403$ eng.pdf

3. E.A. Ashley, Dhorda M, Fairhurst RM, Amaratunga C, Lim P, Suon S, et al. Spread of Artemisinin Resistance in Plasmodium falciparum Malaria. N Engl J Med. 2014;371:411-23.

4. Khattak A A, Venkatesan $M$, Nadeem $M$, et al. Prevalence and distribution of human Plasmodium infection in Pakistan. Malaria J. 2013; 12:297.

5. Murtaza G, Memon IA, Memon AR, Lal MN, Kallar NA. Malaria morbidity in Sindh and the plasmodium species distribution. Pakistan J Med Sci. 2009;25(4):646-9.

6. Cohen JM, Smith DL, Cotter C, et al. Malaria resurgence: a systematic review and assessment of its causes. Malaria J. 2012;11(1):122.

7. Gething PW, Elyazar I, Moyes C, et al. A long neglected world malaria map: Plasmodium vivax endemicity in 2010. PLoS Negl Trop. 2012; 6 (9):e1814.

8. Price RN, von Seidlein L, Valecha N, Nosten F, Baird JK, White NJ. Global extent of chloroquineresistant Plasmodium vivax: A systematic review and meta-analysis. Lancet Infect Dis. 2014;14 (10):982-91.

9. Naing C, Racloz V, Whittaker M, Aung K, Reid SA, Mak JW, et al. Efficacy and safety of dihydroartemisinin-piperaquine for treatment of Plasmodium vivax malaria in endemic countries: meta-analysis of randomised controlled studies. Plos One. 2013; 8(12):e78819.

10. Rodríguez JC, Uribe GÁ, Araújo RM, Narváez PC, Valencia SH. Epidemiology and control of malaria in Colombia. Mem Inst Oswaldo Cruz. 2011;106 
(Suppl-1):114-22.

11. Bloland $P$, Ringwald $P$, Snow R(WHO). Assessment and monitoring of antimalarial drug efficacy for the treatment of uncomplicated falciparum malaria. 2003; World health Organization. Geneva. Availabale from: https:// www.cdc.gov/ malaria/resources/ pdf/ drug_resistance/ who2003_monitoring.pdf

12. WHO. World Malaria Report. World Health Organization. 2016. Available from: http:// www.who.int/malaria/publications/world-malariareport-2016/report/en/

13. Ubben D, Poll EM. MMV in partnership: the Eurartesim® experience. Malaria J. 2013; 12 (1):211.

14. Wells TN, Hooft van Huijsduijnen R, Van Voorhis WC. Malaria medicines: a glass half full? Nat Rev Drug Discov. 2015;14(6):424-42.

15. Awab G, Pukrittayakamee S, Imwong $M$, et al. Dihydroartemisinin-piperaquine versus chloro- quine to treat vivax malaria in Afghanistan: an open randomized, non-inferiority, trial. Malaria $\mathrm{J}$. 2010; 9:105.

16. Pasaribu A, Chokejindachai W. A randomized comparison of dihydroartemisinin-piperaquine and artesunate-amodiaquine combined with primaquine for radical treatment of vivax malaria in sumatera, Indonesia . J Infect Disc. 2013; 208 (11):1906-13.

17. Karunajeewa $\mathrm{H}$, Mueller I, Senn M, et al. A trial of combination antimalarial therapies in children from Papua New Guinea. New Engl J Med 2008; 359 (24):2545-57.

18. Hurtado EK, Claussen AH, Scott KG. Early childhood anemia and mild or moderate mental retardation. Am J Clin Nutr. 1999; 69(1)115-9.

19. Song $S$, Song WO. National nutrition surveys in Asian countries: surveillance and monitoring efforts to improve global health. Asia Pac J Clin Nutr. 2014; 23(4):514-23.

AUTHOR AFFILIATION:
Prof. Salma Shaikh
Department of Pediatrics
Liaquat University of Medical \& Health Sciences
(LUMHS), Hyderabad/Jamshoro, Sindh-Pakistan.
Dr. Imran Ahmed
Assistant Professor, Department of Pediatrics
LUMHS, Hyderabad/Jamshoro, Sindh-Pakistan.
Dr. Sikander Munir Memon (Corresponding Author)
PhD Scholar, Medical Research Center
LUMHS, Jamshoro, Sindh-Pakistan.
Email: drsikandermemon@gmail.com
Dr. Abdul Saleem
Research officer, Department of Pediatrics
LUMHS, Hyderabad/Jamshoro, Sindh-Pakistan.
Dr. Ayaz Babar
Research officer, Department of Pediatrics
LUMHS, Hyderabad/Jamshoro, Sindh-Pakistan.
Research officer, Department of Pediatrics
LUMHS, Hyderabad/Jamshoro, Sindh-Pakistan.
Dreallah Memon

